\title{
Dynamic Behaviors of a Nonautonomous Impulsive Competitive System with the Effect of Toxic Substance
}

\author{
Lijuan Chen $\mathbb{D}^{1},{ }^{1}$ Fengde Chen $\mathbb{D}{ }^{1},{ }^{1}$ and Liujuan Chen $^{2}$ \\ ${ }^{1}$ College of Mathematics and Computer Science, Fuzhou University, Fuzhou, Fujian 350108, China \\ ${ }^{2}$ Department of Science Training, Fujian Institute of Education, Fuzhou, Fujian 350025, China \\ Correspondence should be addressed to Lijuan Chen; chenlijuan@fzu.edu.cn
}

Received 15 April 2018; Revised 11 July 2018; Accepted 26 July 2018; Published 8 August 2018

Academic Editor: Josef Diblík

Copyright (c) 2018 Lijuan Chen et al. This is an open access article distributed under the Creative Commons Attribution License, which permits unrestricted use, distribution, and reproduction in any medium, provided the original work is properly cited.

\begin{abstract}
We firstly propose a nonautonomous impulsive Lotka-Volterra competitive system with the effect of toxic substance. Only one of the two species could produce toxic substance. Sufficient condition which guarantees the extinction of one of the species and the global attractivity of the other species is obtained. We also present an example to verify our main results, which show that species still is possibly driven to extinction when only one of the two species produces toxic substances. The results of this paper supplement the existing results.
\end{abstract}

\section{Introduction}

As we know, investigating the effect of toxic substances on ecological communities is significant. More and more researchers are engaged in this aspect and have obtained lots of excellent results. Smith [1] firstly introduced the effects of toxic substances into a two-species Lotka-Volterra competitive system under an assumption that each species produces a toxic substance to the other only when the other is present. Chattopadhyay [2] obtained a set of sufficient conditions which ensure the system admits a unique globally stable positive equilibrium by constructing a suitable Lyapunov function. Recently, a developing number of scholars pay attention to the effects of toxic substances on competitive model and many distinguished results have been given(see [3-9] and the references cited therein). For example, Chen et al. [3] and Xie et al. [4] considered the global stability of the interior equilibrium point of the competition model of plankton allelopathy. The authors [5-9] discussed the extinction for the competitive model with the effect of toxic substances.

However, we notice that all the above [1-10] only pay attention to the continuous or discrete system. In fact, the actual real system is easily disturbed by human exploring activities such as planting and harvesting. Different from the continuous or discrete systems, the theory of impulsive differential equation is much richer and has a wider application when analyzing many real-world phenomena [11, 12]. Recently, various kinds of systems with impulse have attracted a lot of attention [13-19], to name just a few. Chen et al. [20] considered the extinction property of the following impulsive competitive system with toxic substance:

$$
\begin{aligned}
& \dot{x}_{1}(t)=x_{1}(t)\left(b_{1}(t)-a_{11}(t) x_{1}(t)-a_{12}(t) x_{2}(t)\right. \\
& \left.\quad-d_{1}(t) x_{1}(t) x_{2}(t)\right) \\
& \dot{x}_{2}(t)=x_{2}(t)\left(b_{2}(t)-a_{21}(t) x_{1}(t)-a_{22}(t) x_{2}(t)\right. \\
& \left.\quad-d_{2}(t) x_{1}(t) x_{2}(t)\right) \\
& \quad t \neq \tau_{k}, k \in N, \\
& x_{1}\left(\tau_{k}^{+}\right)=\left(1+h_{1 k}\right) x_{1}\left(\tau_{k}\right), \\
& x_{2}\left(\tau_{k}^{+}\right)=\left(1+h_{2 k}\right) x_{2}\left(\tau_{k}\right),
\end{aligned}
$$

where $x_{i}(t), i=1,2$, denotes the population density of the $i$-th phytoplankton species at time $t$ for a common pool of resources. The terms $d_{1}(t) x_{1}^{2}(t) x_{2}(t)$ and $d_{2}(t) x_{1}(t) x_{2}^{2}(t)$ 
denote the effects of toxic substances which means that each species produces a toxic substance to the other. And $b_{i}(t)$ and $a_{i j}(t), i, j=1,2$, are all continuous functions which are bounded above and below by positive constants. Here the jump conditions reflect the possibility of impulsive effects on the species $x_{1}$ and $x_{2} . h_{i k} x_{i}\left(\tau_{k}\right)<0$ may present the impulsive harvesting amount of the species at $t=\tau_{k}$, while $h_{i k} x_{i}\left(\tau_{k}\right)>0$, the perturbations, may stand for the impulsive stocking amount of the species at $t=\tau_{k}$. That is, the impulsive harvesting amount (or stocking amount) is proportional to the current density of the species.

Given a function $g(t)$, let $g^{L}$ and $g^{M}$ denote inf ${ }_{-\infty<t<+\infty} g(t)$ and $\sup _{-\infty<t<+\infty} g(t)$, respectively.

Also, throughout this paper, the following conditions are assumed.

$\left(A_{1}\right) \quad b_{i}(t), \quad a_{i j}(t)(i, j=1,2), \quad d_{i}(t)$ are continuous $T$-periodic functions such that $b_{i}^{L}>0, a_{i j}^{L}>0, d_{i}^{L}>0$ and $N=\{1,2, \ldots\}$.

$\left(A_{2}\right)\left\{\tau_{k}\right\}_{k \in N}$ satisfies $0=\tau_{0}<\tau_{1}<\tau_{2}<\cdots<\tau_{k}<\cdots$ and $\lim _{k \rightarrow \infty} \tau_{k}=+\infty$.

$\left(A_{3}\right) h_{i k}(i=1,2, k \in N)$ are constants and there exists an integer $q>0$ such that $h_{i(k+q)}=h_{i k}, \tau_{k+q}=\tau_{k}+T$. A natural constraint in this case is $1+h_{i k}>0, i=1,2, k \in N$.

$\left(A_{4}\right) \overline{b_{1}^{L}}=b_{1}^{L}+(1 / T) \sum_{k=1}^{q} \ln \left(1+h_{1 k}\right)>0$ and $\overline{b_{2}^{M}}=$ $b_{2}^{M}+(1 / T) \sum_{k=1}^{q} \ln \left(1+h_{2 k}\right)>0$.

Chen et al.[20] proved that if the coefficients of system (1) satisfy

$$
\begin{aligned}
& \overline{b_{1}^{L}} a_{22}^{L}>\overline{b_{2}^{M}} a_{12}^{M}, \\
& \overline{b_{1}^{L}} a_{21}^{L} \geq \overline{b_{2}^{M}} a_{11}^{M}, \\
& \overline{b_{1}^{L}} d_{2}^{L} \geq \overline{b_{2}^{M}} d_{1}^{M},
\end{aligned}
$$

then the species $x_{2}$ of system (1) will be driven to extinction while the species $x_{1}$ will be globally attractive with any positive solution of an impulsive logistic equation.

In [21], the author considered the following LotkaVolterra type of model for two interesting phytoplankton species:

$$
\begin{aligned}
& \dot{x}_{1}(t) \\
& \quad=x_{1}(t)\left(r_{1}-a_{1} x_{1}(t)-b_{1} x_{2}(t)-c_{1} x_{1}(t) x_{2}(t)\right), \\
& \dot{x}_{2}(t)=x_{2}(t)\left(r_{2}-a_{2} x_{1}(t)-b_{2} x_{2}(t)\right) .
\end{aligned}
$$

Since many biological or environmental parameters are subject to fluctuate with time, a plausible mathematical model may take the following form:

$$
\begin{aligned}
& \dot{x}_{1}(t)=x_{1}(t)\left(r_{1}(t)-a_{1}(t) x_{1}(t)-b_{1}(t) x_{2}(t)\right. \\
& \left.\quad-c_{1}(t) x_{1}(t) x_{2}(t)\right) \\
& \dot{x}_{2}(t)=x_{2}(t)\left(r_{2}(t)-a_{2}(t) x_{1}(t)-b_{2}(t) x_{2}(t)\right) .
\end{aligned}
$$

To the best of the authors' knowledge, until this day, there exist no results which focus on the dynamic behaviors of the above system with impulse. All the facts stated above motivate us to investigate the extinction problem for a Lotka-Volterra competitive system with impulse and one toxin producing phytoplankton, i.e., the following system:

$$
\begin{aligned}
& \dot{x}_{1}(t)=x_{1}(t)\left(b_{1}(t)-a_{11}(t) x_{1}(t)-a_{12}(t) x_{2}(t)\right. \\
& \left.\quad-d_{1}(t) x_{1}(t) x_{2}(t)\right) \\
& \dot{x}_{2}(t)=x_{2}(t)\left(b_{2}(t)-a_{21}(t) x_{1}(t)-a_{22}(t) x_{2}(t)\right) \\
& \quad t \neq \tau_{k}, k \in N, \\
& x_{1}\left(\tau_{k}^{+}\right)=\left(1+h_{1 k}\right) x_{1}\left(\tau_{k}\right), \\
& x_{2}\left(\tau_{k}^{+}\right)=\left(1+h_{2 k}\right) x_{2}\left(\tau_{k}\right) .
\end{aligned}
$$

with initial condition

$$
\begin{aligned}
& x_{1}(0)>0, \\
& x_{2}(0)>0 .
\end{aligned}
$$

Here $x_{1}(t)$ denotes the density of nontoxic phytoplankton. In other words, the first species could not produce toxic substance since $d_{2}(t)=0$. And the second species $x_{2}(t)$ is toxic liberating phytoplankton which could produce toxic substance due to $d_{1}(t) \neq 0$. And all the coefficients in the above system have the same restriction as that of system (1). Comparing (4) with (1), one could find that (4) is the special case of (1), i.e., $d_{2}(t)=0$. Hence, one may conjecture that the results of [20] could be applied directly to system (4) in this paper. However, unfortunately, one can notice that the inequality

$$
\overline{b_{1}^{L}} d_{2}^{L} \geq \overline{b_{2}^{M}} d_{1}^{M}
$$

in $\left(H_{1}\right)$ no longer holds since $d_{2}(t)=0$. As a result, for $(4)$, it is very necessary to figure out a new sufficient condition to guarantee the extinction of the species $x_{2}$ and the global attractivity of the species $x_{1}$. The new sufficient condition which will be presented in the next section is different from $\left(H_{1}\right)$. And the obtained results in the next section will be a good complement of those in [20].

By the basic theories of impulsive differential equations in $[22,23]$, system (4) has a unique solution $x(t)$ $=x\left(t, t_{0}\right) \in P C\left([0,+\infty), R^{2}\right)$ and $P C\left([0,+\infty), R^{2}\right)=\{\phi:[0$, $+\infty) \longrightarrow R^{2}, \phi$ is continuous for $t \neq \tau_{k}$. Also $\phi\left(\tau_{k}^{-}\right)$ and $\phi\left(\tau_{k}^{+}\right)$exist, and $\left.\phi\left(\tau_{k}^{-}\right)=\phi\left(\tau_{k}^{+}\right), k \in N\right\}$ for each initial value $x(0)=x_{0} \in R^{2+}$.

The organization of this paper is as follows. In Section 2, necessary preliminaries are presented. In Section 3, we give the sufficient conditions ensuring the extinction and global attractivity of the system. In Section 4, numerical simulations are presented to illustrate the feasibility of our main results. In the last section, we give a brief discussion.

\section{Lemmas}

In this section, we state the following lemmas [20] which will be useful in the proof of our main results. 
Lemma 1. Assume that $m \in P C\left[R_{+}, R\right]$ with points of discontinuity at $t=\tau_{k}$ and is left continuous at $t=\tau_{k}, k \in N$, and that

$$
\begin{aligned}
D^{+} m(t) & \leq g(t, m(t)), \quad t \neq \tau_{k}, \\
m\left(\tau_{k}^{+}\right) & \leq \phi_{k}\left(m\left(\tau_{k}\right)\right),
\end{aligned}
$$

where $g \in C\left[R_{+} \times R_{+}, R\right], \phi_{k} \in C[R, R]$ and $\phi_{k}(u)$ is nondecreasing in $u$ for each $k \in N$. Let $r(t)$ be the maximal solution of the scalar impulsive differential equation:

$$
\begin{aligned}
\dot{u}(t) & =g(t, u(t)), \quad t \neq \tau_{k}, \\
u\left(\tau_{k}^{+}\right) & =\phi_{k}\left(u\left(\tau_{k}\right)\right) \geq 0, \quad \tau_{k}>t_{0}, k \in N, \\
u\left(t_{0}^{+}\right) & =u_{0},
\end{aligned}
$$

existing on $\left[t_{0}, \infty\right)$, then $m\left(t_{0}^{+}\right) \leq u_{0}$ implies $m(t) \leq r(t), t \geq$ $t_{0}$.

Lemma 2. Consider the following impulsive system:

$$
\begin{aligned}
\dot{x}(t) & =x(t)(a(t)-b(t) x(t)), \quad t \neq \tau_{k}, \\
x\left(\tau_{k}^{+}\right) & =\left(1+c_{k}\right) x\left(\tau_{k}\right), \quad k \in N,
\end{aligned}
$$

where $a(t), b(t)$ are continuous $T$-periodic functions and $b(t)>0$, and there exists an integer $q>0$ such that $c_{k+q}=$ $c_{k}, t_{k+q}=t_{k}+T$ and $c_{k}>-1$ for $k \in N$. Then (9) has a unique positive $T$-periodic solution $\theta_{[a, b]}$ if and only if

$$
\prod_{k=1}^{q}\left(1+c_{k}\right) \exp \left\{\int_{0}^{T} a(t) d t\right\}>1
$$

which is equivalent to the inequality $\int_{0}^{T} a(t) d t+\sum_{k=1}^{q} \ln (1+$ $\left.c_{k}\right)>0$. Moreover, the unique positive $T$-periodic solution $\theta_{[a, b]}$ is globally asymptotically stable.

Lemma 3. Let $X(t)=\left(x_{1}(t), x_{2}(t)\right)^{T}$ be any solution of system (4) such that $x_{i}\left(0^{+}\right)>0$, then $x_{i}(t)>0$ for all $t \geq 0$.

Lemma 4. Let $X(t)=\left(x_{1}(t), x_{2}(t)\right)^{T}$ be any solution of system (4) such that $x_{i}\left(0^{+}\right)>0, i=1,2$, then we have

$$
\limsup _{t \rightarrow+\infty} x_{i}(t) \leq \theta_{\left[b_{i}, a_{i i}\right]}^{M}, \quad i=1,2
$$

where $\theta_{\left[b_{i}, a_{i i}\right]}$ is the unique positive solution of the following Logistic equation with impulse

$$
\begin{aligned}
x^{\prime}(t) & =x(t)\left(b_{i}(t)-a_{i i}(t) x(t)\right), \quad t \neq \tau_{k}, k \in N, \\
x\left(\tau_{k}^{+}\right) & =\left(1+h_{i k}\right) x\left(\tau_{k}\right),
\end{aligned}
$$

\section{Main Results}

In this section, we present our main results for system (4). In the following, we firstly present the extinction of the species $x_{2}$.

Theorem 5. If there exists $\rho \in[0,1]$ such that the following condition

$$
\begin{aligned}
& \overline{b_{1}^{L}} a_{22}^{L}>\overline{b_{2}^{M}}\left(a_{12}^{M}+\rho d_{1}^{M} \theta_{\left[b_{1}, a_{11}\right]}^{M}\right), \\
& \overline{b_{1}^{L}} a_{21}^{L}>\overline{b_{2}^{M}}\left(a_{11}^{M}+(1-\rho) d_{1}^{M} \theta_{\left[b_{2}, a_{22}\right]}^{M}\right)
\end{aligned}
$$

holds, then any positive solution $\left(x_{1}(t), x_{2}(t)\right)^{T}$ of system (4) satisfies

$$
\lim _{t \rightarrow+\infty} x_{2}(t)=0
$$

Proof. By $\left(\mathrm{H}_{2}\right)$, we can choose positive constants $\gamma$ and $\eta$ such that

$$
\begin{aligned}
& \frac{\overline{b_{1}^{L}}}{\overline{b_{2}^{M}}}>\frac{\eta}{\gamma}>\frac{a_{12}^{M}+\rho d_{1}^{M} \theta_{\left[b_{1}, a_{11}\right]}^{M}}{a_{22}^{L}}, \\
& \frac{\overline{b_{1}^{L}}}{\overline{b_{2}^{M}}}>\frac{\eta}{\gamma}>\frac{a_{11}^{M}+(1-\rho) d_{1}^{M} \theta_{\left[b_{2}, a_{22}\right]}^{M}}{a_{21}^{L}} \text {. }
\end{aligned}
$$

So from Lemma 4 , there exist positive constants $\varepsilon$, $T_{0}$ such that $x_{i}(t) \leq \theta_{\left[b_{i}, a_{i i}\right]}+\varepsilon$ and

$$
\begin{array}{r}
\eta a_{22}(t)-\gamma\left(a_{12}(t)+\rho d_{1}(t)\left(\theta_{\left[b_{1}, a_{11}\right]}+\varepsilon\right)\right)>0, \\
\eta a_{21}(t)-\gamma\left(a_{11}(t)+(1-\rho) d_{1}(t)\left(\theta_{\left[b_{2}, a_{22}\right]}+\varepsilon\right)\right)>0
\end{array}
$$

when $t \geq T_{0}$. Also there exists $\delta>0$ such that

$$
\begin{aligned}
& \eta\left(b_{2}(t)+\frac{1}{T} \sum_{k=1}^{q} \ln \left(1+h_{2 k}\right)\right) \\
& \quad-\gamma\left(b_{1}(t)+\frac{1}{T} \sum_{k=1}^{q} \ln \left(1+h_{1 k}\right)\right)<-\frac{\delta}{T}<0 .
\end{aligned}
$$

From system (4) and (15), we have

$$
\begin{aligned}
& \frac{d}{d t}\left[\ln \frac{\left(x_{2}(t)\right)^{\eta}}{\left(x_{1}(t)\right)^{\gamma}}\right]=\left(\eta b_{2}(t)-\gamma b_{1}(t)\right)-\left(\eta a_{22}(t)\right. \\
& \left.\quad-\gamma a_{12}(t)\right) x_{2}(t)-\left(\eta a_{21}(t)-\gamma a_{11}(t)\right) x_{1}(t) \\
& \quad+\gamma d_{1}(t) x_{1}(t) x_{2}(t) \leq\left(\eta b_{2}(t)-\gamma b_{1}(t)\right) \\
& \quad-\left(\eta a_{22}(t)-\gamma a_{12}(t)-\gamma \rho d_{1}(t)\left(\theta_{\left[b_{1}, a_{11}\right]}+\varepsilon\right)\right) \\
& \quad x_{2}(t)-\left(\eta a_{21}(t)-\gamma a_{11}(t)\right. \\
& \left.\quad-\gamma(1-\rho) d_{1}(t)\left(\theta_{\left[b_{2}, a_{22}\right]}+\varepsilon\right)\right) x_{1}(t) \leq \eta b_{2}(t) \\
& \quad-\gamma b_{1}(t), \quad t \neq \tau_{k}, t>T_{0}
\end{aligned}
$$


and

$$
\begin{aligned}
\ln \left(\frac{\left(x_{2}\left(\tau_{k}^{+}\right)\right)^{\eta}}{\left(x_{1}\left(\tau_{k}^{+}\right)\right)^{\gamma}}\right)= & \ln \left(\frac{\left(1+h_{2 k}\right)^{\eta}}{\left(1+h_{1 k}\right)^{\gamma}}\right) \\
& +\ln \left(\frac{\left(x_{2}\left(\tau_{k}\right)\right)^{\eta}}{\left(x_{1}\left(\tau_{k}\right)\right)^{\gamma}}\right) .
\end{aligned}
$$

Integrating both sides of (17) over internals $\left[T_{0}, T_{0}+\tau_{1}\right),\left[T_{0}+\right.$ $\left.\tau_{1}, T_{0}+\tau_{2}\right),\left[T_{0}+\tau_{2}, T_{0}+\tau_{3}\right),\left[T_{0}+\tau_{\sigma-1}, T_{0}+\tau_{\sigma}\right)$ and $\left[T_{0}+\tau_{\sigma}, t\right)$, respectively, and adding the $\sigma$ inequalities, for $t \in\left[T_{0}+\tau_{\sigma}, T_{0}+\right.$ $\left.\tau_{\sigma+1}\right)$ and $T_{0}+\tau_{\sigma} \in\left[m_{1} T,\left(m_{1}+1\right) T\right), m_{1} \in N$, we obtain

$$
\begin{aligned}
\ln \left(\frac{\left(x_{2}(t)\right)^{\eta}}{\left(x_{1}(t)\right)^{\gamma}}\right)-\ln \left(\frac{\left(x_{2}\left(T_{0}\right)\right)^{\eta}}{\left(x_{1}\left(T_{0}\right)\right)^{\gamma}}\right) \\
<\int_{T_{0}}^{t}\left(\eta b_{2}(t)-\gamma b_{1}(t)\right) d t+\ln \frac{\prod_{T_{0}<\tau_{k}<t}\left(1+h_{2 k}\right)^{\eta}}{\prod_{T_{0}<\tau_{k}<t}\left(1+h_{1 k}\right)^{\gamma}} \\
=m_{1} \int_{0}^{T}\left(\eta b_{2}(t)-\gamma b_{1}(t)\right) d t \\
\quad+m_{1} \eta \sum_{k=1}^{q} \ln \left(1+h_{2 k}\right)-m_{1} \gamma \sum_{k=1}^{q} \ln \left(1+h_{1 k}\right) \\
\quad+\int_{m_{1} T}^{t}\left(\eta b_{2}(t)-\gamma b_{1}(t)\right) d t \\
\quad+\ln \frac{\prod_{m_{1} T<\tau_{k}<t}\left(1+h_{2 k}\right)^{\eta}}{\prod_{m_{1} T<\tau_{k}<t}\left(1+h_{1 k}\right)^{\gamma}} \\
\leq m_{1} \eta\left(T b_{2}^{M}+\sum_{k=1}^{q} \ln \left(1+h_{2 k}\right)\right) \\
\quad-m_{1} \gamma\left(T b_{1}^{L}+\sum_{k=1}^{q} \ln \left(1+h_{1 k}\right)\right)+B \\
\leq-m_{1} \delta+B,
\end{aligned}
$$

where $B=\max _{0 \leq s \leq T}\left(\int_{0}^{s}\left(\eta b_{2}(t)-\gamma b_{1}(t)\right) d t+\ln \left(\prod_{0 \leq \tau_{k}<s}(1+\right.\right.$ $\left.\left.\left.\left.h_{2 k}\right)^{\eta}\right) / \prod_{0 \leq \tau_{k}<s}\left(1+h_{1 k}\right)^{y}\right)\right)$. This shows that

$$
\left(x_{2}(t)\right)^{\eta} \leq\left(x_{1}(t)\right)^{\gamma} \exp \left(-m_{1} \delta+B\right) \frac{\left(x_{2}\left(T_{0}\right)\right)^{\eta}}{\left(x_{1}\left(T_{0}\right)\right)^{\gamma}} .
$$

According to Lemma 4 , we notice that $x_{1}(t)$ is ultimately upper bounded; hence, we obtain $\lim _{t \rightarrow+\infty} x_{2}(t)=0$. This completes the proof of Theorem 5 .

In the above, we have provided the sufficient condition to ensure that species $x_{2}$ is extinct. Similar to the proof of Theorems 3.2 and 3.3 in [20], we can also obtain the following result about the global attractivity of the species $x_{1}$.

Theorem 6. Assume that $\left(H_{2}\right)$ holds, then the species $x_{1}$ is globally attractive; i.e., for any positive solution $\left(x_{1}(t), x_{2}(t)\right)^{T}$ of system (4) and any positive solution $x(t)$ of the impulsive logistic equation,

$$
\begin{aligned}
\dot{x}(t) & =x(t)\left(b_{1}(t)-a_{11}(t) x(t)\right), \quad t \neq \tau_{k}, k \in N, \\
x\left(\tau_{k}^{+}\right) & =\left(1+h_{1 k}\right) x\left(\tau_{k}\right),
\end{aligned}
$$

one has

$$
\lim _{t \rightarrow+\infty}\left(x_{1}(t)-x(t)\right)=0 .
$$

Remark 7. For system (4), condition $\left(H_{1}\right)$ is not satisfied due to $d_{2}(t)=0$. As a result, we cannot easily deduce the extinction of the species $x_{2}$ and the globally attractivity of the species $x_{1}$ from Theorem 3.1-3.3 in [20]. However, in Theorems 5-6 of this paper, we have obtained the sufficient condition $\left(\mathrm{H}_{2}\right)$ under which the corresponding dynamic behaviors for (4) are presented. Also we can find that conditions $\left(\mathrm{H}_{1}\right)$ and $\left(\mathrm{H}_{2}\right)$ cannot be derived from each other. In other words, the above two conditions are complementary. Thus, the main results in this paper are indeed a good complement of those in [20].

Remark 8. According to the main results in [20], for system (4), one can easily induce the extinction of the species $x_{1}$ and the global attractivity of the species $x_{2}$ under the condition $\overline{b_{2}^{L}} a_{12}^{L}>\overline{b_{1}^{M}} a_{22}^{M}, \overline{b_{2}^{L}} a_{11}^{L} \geq \overline{b_{1}^{M}} a_{21}^{M}$. This paper pays attention to figure out a new sufficient condition to guarantee the extinction of the species $x_{2}$ and the global attractivity of the species $x_{1}$. The obtained results show that the species $x_{2}$ can still be extinct even though the species $x_{1}$ do not produce toxic substance.

\section{Example}

In this section, we give numerical simulation to illustrate the main theoretical results above.

Example 1. In system (4), let $b_{1}(t)=1.4+0.2 \sin (4 \pi t)$, $a_{11}(t)=0.5, a_{12}(t)=0.2, d_{1}(t)=0.4, h_{1 k}=\exp (1)-$ $1, b_{2}(t)=0.7+0.4 \sin (4 \pi t), a_{21}(t)=0.5, a_{22}(t)=1.3, h_{2 k}=$ $\exp (1 / 2)-1$. By computing, we derive $\theta_{\left[b_{1}, a_{11}\right]}^{M} \leq 12, \theta_{\left[b_{2}, a_{22}\right]}^{M} \leq$ 1.8 and, choosing $\rho=0.4$, we have $\overline{b_{1}^{L}} a_{22}^{L}=4.42, \overline{b_{2}^{M}}\left(a_{12}^{M}+\right.$ $\left.\rho d_{1}^{M} \theta_{\left[b_{1}, a_{11}\right]}^{M}\right) \leq 3.604, \overline{b_{2}^{M}}\left(a_{11}^{M}+(1-\rho) d_{1}^{M} \theta_{\left[b_{2}, a_{22}\right]}^{M}\right) \leq 1.5844$. So the condition $\left(H_{2}\right)$ holds. From Theorems 5-6, it follows that species $x_{2}$ is extinct while species $x_{1}$ is globally stable (see Figure 1).

\section{Conclusion}

In this paper, a nonautonomous impulsive Lotka-Volterra competitive system with the effect of toxic substance has been studied. We have obtained the sufficient condition which guarantees one of the species' extinction and the other species' global attractivity. The results in this paper supplement those in [20]. We also have presented an example to verify our main results, which show that the species can still be driven to extinction when only one of the two species produces toxic substance. 




(a) Species $x_{1}$ is permanent

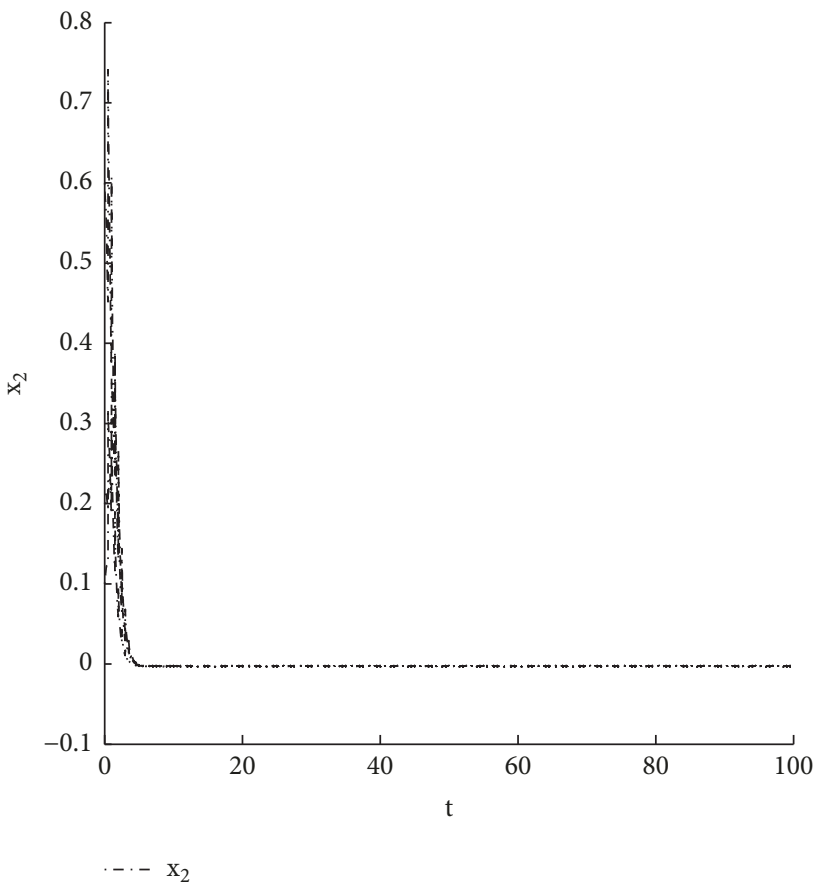

(b) Species $x_{2}$ is extinct

FIGURE 1: Dynamic behaviors of system (4) with the initial conditions $(0.1,0.1)^{T},(1,0.6)^{T}$, and $(0.8,0.2)^{T}$.

\section{Data Availability}

The data used to support the findings of this study are available from the corresponding author upon request.

\section{Conflicts of Interest}

The authors declare that they have no conflicts of interest.

\section{Acknowledgments}

This work was supported by the National Natural Science Foundation of China under Grant 11601085, the Natural Science Foundation of Fujian Province (2017J01400), and the Scientific Research Foundation of Fuzhou University (GXRC-17026).

\section{References}

[1] J. Maynard-Smith, Models in Ecology, Cambridge University Press, Cambridge, UK, 1975.

[2] J. Chattopadhyay, "Effect of toxic substances on a two-species competitive system," Ecological Modelling, vol. 84, no. 1-3, pp. 287-289, 1996.

[3] F. D. Chen, X. D. Xie, and H. N. Wang, "Global stability in a competition model of plankton allelopathy with infinite delay," Journal of Systems Science and Complexity, vol. 28, no. 5, pp. 1070-1079, 2015.

[4] X. Xie, Y. Xue, and R. Wu, "Global attractivity of a discrete competition model of plankton allelopathy with infinite deviating arguments," Advances in Difference Equations, Article ID 303, 2016.

[5] Q. Yue, "Extinction for a discrete competition system with the effect of toxic substances," Advances in Difference Equations, Article ID 1, 2016.

[6] X. Xie, Y. Xue, R. Wu, and L. Zhao, "Extinction of a two species competitive system with nonlinear inter-inhibition terms and one toxin producing phytoplankton," Advances in Difference Equations, Paper No. 258, 13 pages, 2016.

[7] F. Chen, X. Xie, Z. Miao, and L. Pu, "Extinction in two species nonautonomous nonlinear competitive system," Applied Mathematics and Computation, vol. 274, no. 1, pp. 119-124, 2016.

[8] F. Chen, X. Chen, and S. Huang, "Extinction of a two species non-autonomous competitive system with BeddingtonDeAngelis functional response and the effect of toxic substances," Open Mathematics, vol. 14, pp. 1157-1173, 2016.

[9] L. J. Chen and F. D. Chen, "Extinction in a discrete LotkaVolterra competitive system with the effect of toxic substances and feedback controls," International Journal of Biomathematics, vol. 8, no. 1, Article ID 1550012, 13 pages, 2015.

[10] K. Yang, Z. S. Miao, F. D. Chen, and X. D. Xie, "Influence of single feedback control variable on an autonomous Holling-II type cooperative system," Journal of Mathematical Analysis and Applications, vol. 435, no. 1, pp. 874-888, 2016.

[11] A. Halanay and D. Wexler, Qualitative Theory of Systems with Impulses, Nauka, Moscow, 1971.

[12] A. M. Samoilenko and N. A. Perestyuk, Impulsive Differential Equations, World Scientific, Singapore, 1995.

[13] L. Stone, B. Shulgin, and Z. Agur, "Theoretical examination of the pulse vaccination policy in the SIR epidemic model," Mathematical and Computer Modelling, vol. 31, no. 4-5, pp. 207$215,2000$. 
[14] B. Shulgin, L. Stone, and Z. Agur, "Pulse vaccination strategy in the SIR epidemic model," Bulletin of Mathematical Biology, vol. 60, no. 6, pp. 1123-1148, 1998.

[15] A. d'Onofrio, "On pulse vaccination strategy in the SIR epidemic model with vertical transmission," Applied Mathematics Letters, vol. 18, no. 7, pp. 729-732, 2005.

[16] S. Nundloll, L. Mailleret, and F. Grognard, "Two models of interfering predators in impulsive biological control," Journal of Biological Dynamics, vol. 4, no. 1, pp. 102-114, 2010.

[17] L. Chen and F. Chen, "Dynamic behaviors of the periodic predator-prey system with distributed time delays and impulsive effect," Nonlinear Analysis: Real World Applications, vol. 12, no. 4, pp. 2467-2473, 2011.

[18] L. J. Chen and J. T. Sun, "Distributed optimal analysis for the multi-agent system with hybrid protocols," Journal of The Franklin Institute, vol. 354, no. 2, pp. 1160-1168, 2017.

[19] L. J. Chen and J. T. Sun, "Distributed optimal control and L2 gain performance for the multi-agent system with impulsive effects," Systems and Control Letters, vol. 113, pp. 65-70, 2018.

[20] L. Chen, J. Sun, F. Chen, and L. Zhao, "Extinction in a LotkaVolterra competitive system with impulse and the effect of toxic substances," Applied Mathematical Modelling: Simulation and Computation for Engineering and Environmental Systems, vol. 40, no. 3, pp. 2015-2024, 2016.

[21] J. Solé, E. García-Ladona, P. Ruardij, and M. Estrada, "Modelling allelopathy among marine algae," Ecological Modelling, vol. 183, no. 4, pp. 373-384, 2005.

[22] V. Lakshmikantham, D. D. Bainov, and P. S. Simeonov, Theory of Impulsive Differential Equations, World Scientific, Singapore, 1989.

[23] D. Bainov and P. Simeonov, Impulsive Differential Equations: Periodic Solutions and Applications, Longman Scientific and Technical, New York, NY, USA, 1993. 


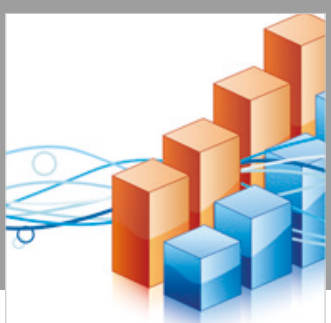

Advances in

Operations Research

\section{-n-m}
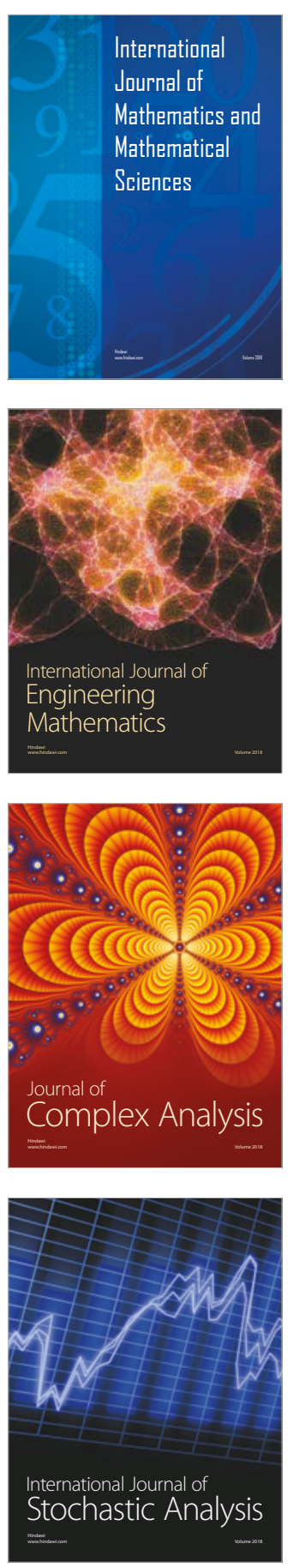


Journal of

Applied Mathematics


Submit your manuscripts at

www.hindawi.com

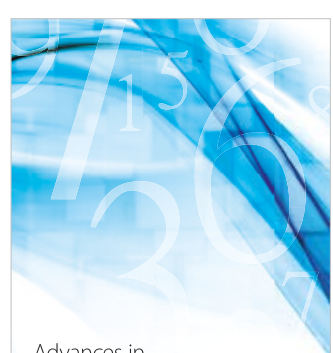

Advances in
Numerical Analysis
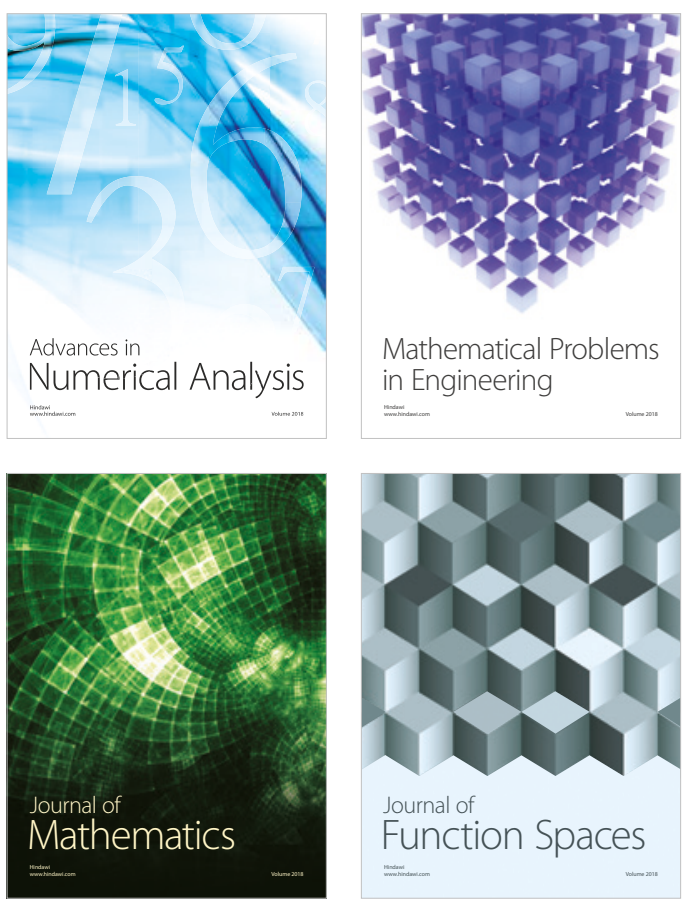

Mathematical Problems in Engineering

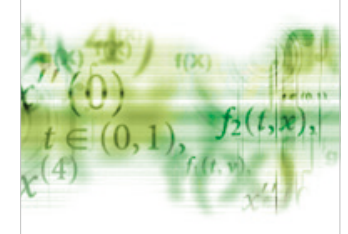

International Journal of

Differential Equations



Journal of

Function Spaces

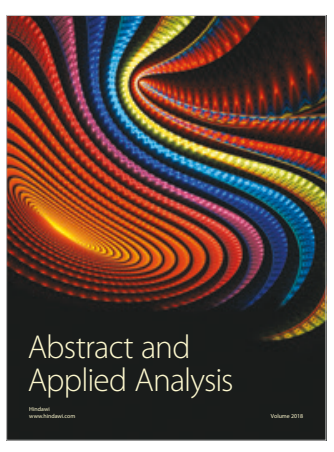

The Scientific

World Journal

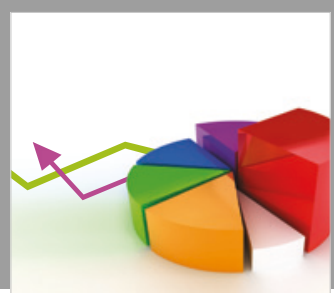

Journal of

Probability and Statistics
